\title{
How Serious is the Economic Burden of Diabetes Mellitus in Hainan Province? A Study Based on "System of Health Account 2011"
}

\author{
Yuanyuan Dong • Chunping Liu · Peng Zhou • Yalan Zhu • \\ Qingcheng Tang · Siyu Wang · Xin Wang (D)
}

Received: September 6, 2019 / Published online: October 18, 2019

(c) The Author(s) 2019

\begin{abstract}
Introduction: The treatment of diabetes requires extensive use of healthcare resources, resulting in high medical costs, which in turn places a heavy economic burden on society, patients and their families.

Methods: A multi-stage stratified random sampling method was used to investigate 283 medical and health institutions in Hainan
\end{abstract}

Yuanyuan Dong and Chunping Liu are co-first authors.

Enhanced Digital Features To view enhanced digital features for this article go to https://doi.org/10.6084/ m9.figshare.9948575.

Y. Dong $\cdot$ Y. Zhu $\cdot$ X. Wang $(\bowtie)$

College of the Humanities and Social Sciences, China Medical University, Shenyang, People's

Republic of China

e-mail: wxinsmile@qq.com

C. Liu · P. Zhou

Administration School, Hainan Medical University, Haikou, People's Republic of China

Q. Tang

Medical Information School, Hainan Medical

University, Haikou, People's Republic of China

S. Wang

Clinical Medical Science, China Medical University,

Shenyang, People's Republic of China

X. Wang

School of Public Health, Xinjiang Medical

University, Urumqi, People's Republic of China
Province. The total medical expenses relating to diabetes in Hainan Province in 2016, institutional flow directions, the composition of service functions and the distribution of the healthcare costs to beneficiaries were analyzed based on the System of Health Account 2011. The STATA version 12.0 statistical software package was used to collate operation data, and SPSS software was used to carry out regression analysis on the factors affecting hospitalization costs.

Results: In 2016, the total medical expenses for the treatment of diabetes in Hainan Province was 242.17 billion renminbi (RMB), of which $81.95 \%$ was spent in high-level hospitals and $14.71 \%$ was spent in medical institutions providing primary care. There was little difference between outpatient and hospitalization expenses (53.01 and 46.99\%, respectively). Hospitalization accounted for $77.62 \%$ of the expenses of medical institutions providing primary care. Older patients were found to spend more on medical and drug expenditure.

Conclusion: The economic burden of healthcare expenses for the treatment of diabetes in Hainan Province is massive, and patient treatment is concentrated in large hospitals. It is necessary to inform patients to focus more on medical institutions that provide primary care, adjust the proportion of medical insurance reimbursement, control the cost of hospitalization and strengthen the healthcare management of middle-aged and elderly diabetic 
patients. Only in this way can costs be reduced and the economic burden be eased.

Keywords: Diabetes; Medical expenses; System of Health Account 2011

\section{Key Summary Points}

Why carry out this study?

The treatment of diabetes consumes considerable healthcare resources, resulting in high medical costs, which places a heavy economic burden on society, patients and their families.

The aim of this research was to study, on the scale of medical expenses for diabetic patients in Hainan Province in 2016, the distribution of the expenses of the beneficiaries and the flow direction of institutions, to provide a reference for health departments to formulate health policies and allocate medical resources rationally.

\section{What was learned from the study?}

The medical burden of diabetes treatment in Hainan Province is heavy, and patient treatment is concentrated in large hospitals. It is necessary to inform patients to focus more on medical institutions that provide primary care, adjust the proportion of medical insurance reimbursement, control the cost of hospitalization and strengthen the healthcare management of middle-aged and elderly diabetic patients.

The medical expenses of diabetes treatment mainly flow to high-level medical institutions, and medical institutions providing primary care fail to function fully in their role of "gatekeeper." Early prevention and early treatment of diabetes mellitus will likely be the key to reduce the burden of medical expenses for diabetes mellitus in the future.

\section{INTRODUCTION}

Diabetes mellitus is a group of metabolic diseases characterized by hyperglycemia [1]. With the rapid economic developments and the abrupt transition of lifestyles and society in recent years, morbidity and mortality linked to diabetes are increasing rapidly worldwide [2], with the number of diabetic patients increasing year by year. According to a recent survey, around 415 million adults globally have diabetes, of whom approximately 110 million are in China [3]. China has become the secondlargest country with diabetes after India. Updated data show that the estimated overall prevalence of diabetes and prediabetes in Chinese adults is 10.9 and $35.7 \%$, respectively [4].

Diabetes, accompanied by multiple complications, cannot be cured and requires life-long medication. Patients with diabetes are fourfold more likely to suffer from cerebrovascular disease and heart disease than those without diabetes, and the mortality of a diabetic person with hypertension is sevenfold higher than that of a person with uncomplicated hypertension [5]. The treatment of diabetes requires extensive use of medical resources, resulting in high medical costs. These costs are a heavy economic burden on society, patients and their families alike and have a serious impact on social development at both the individual and population level. Studies have shown that diabetes is beginning to shift to younger age groups, such as teenagers, with this trend growing in recent years. This trend towards rejuvenation is an invisible burden to the family [6]. With the advent of complications, there will also be conflicts between work and treatment, which will also negatively affect the economic burden of the family and society. It is therefore evident that diabetes has become a public health problem that cannot be ignored.

In the study reported here, we have evaluated the economic burden of diabetes patients seeking medical treatment in Hainan Province. We chose Hainan Province because it provides a significant reference point for such studies in China, with average levels of both economic and medical development in comparison to 
other provinces. We also established a comparable database for other provinces, which will allow us to perform similar studies in the future. It must be noted that the study of the disease burden is still in its infancy in Hainan Province, with only a few large-scale and high-quality studies on this subject in Hainan Province [7]. To our knowledge, no research report on the cost of diabetes treatment in Hainan Province has ever been published. In the present study, we studied the scale of medical expenses for diabetic patients in Hainan Province in 2016, the distribution of the expenses of the beneficiaries and the flow direction of institutions, with the aim to provide a reference for health departments to formulate health policies and allocate medical resources rationally.

The System of Health Account 2011 (SHA 2011) is a well-known health cost accounting system worldwide that was formulated by the World Health Organization (WHO), the Organization for Economic Cooperation and Development (OECD) and the European Union Statistical Office (EUROSTAT) [8]. The application of this new accounting system to a study of medical expenses in Hainan Province can bring these expenses better in line with international standards and improve the international and domestic comparability of these expenses in Hainan Province. It can help contribute to modifications of health policies and the allocation of medical resources in Hainan Province.

This overall purposes of this study was to evaluate the economic burden of diabetes patients in seeking medical treatment by calculating the medical cost of diabetes in Hainan Province and to propose countermeasures to improve the medical condition of patients and relieve the economic pressure.

\section{METHODS}

\section{Data Source}

All of the data were derived from the annual report of health statistics of Hainan Province in 2016 and the annual report of health finance of Hainan Province in 2016. The health statistics yearbook and health financial annual report were provided by the Hainan Health Career Development Center. The case data came from 283 medical institutions and public health institutions in Hainan Province through a sampling survey.

\section{Data Sample}

The study used a multistage stratified random sampling method. The first stage was to select the sample cities and counties, taking into account the level of economic development, medical services and medical information management system in Hainan Province, as well as other factors. We chose a prefecture-level city, Haikou, and three counties, including Wenchang (considered to be a county-level city), Chengmai and Dingan. The second stage was to select survey areas. For this purpose, we extracted two districts from Haikou-Qiongshan district and Longhua district-and three towns and three villages from each of the three counties. In the third stage, we selected medical institutions in the selected areas according to the level and type of medical institutions. A total of 283 health institutions were selected, including ten in Hainan Province, eight in Haikou City, 45 in Wenchang City (county), 39 in Qiongshan District, 54 in Longhua District, 73 in Chengmai County and 54 in Dingan County. The data on outpatient and inpatient cases collected from the sample survey institutions were classified on the basis of the medical and health system in Hainan Province. Once all invalid or incorrect data had been removed, the available data encompassed a total of 2,529,946 samples. The basic information included patient's sex, age, disease diagnosis, medical expenses, type of medical insurance payment, type of medical institutions, among other factors. Patients with endocrine, nutritional and metabolic diseases in Hainan Province in 2016 were included in the study. The types of diabetes (type 1 diabetes, type 2 diabetes, malnutritional diabetes) and their complications were studied. Patient information was normalized according to the "International classification of diseases, tenth revision" (ICD-10; https://www. who.int/classifications/icd/en/). The main 
factors influencing hospitalization medical expenses of 12,171 diabetic patients were statistically analyzed.

\section{Statistical Methods}

The accounting principles and methods adopted in the study are according to the revised edition of "A system of health accounts 2011" [8] and are further explained in the following sections.

\section{Accounting of Medical Expenses}

The medical expenses $\left(C_{\mathrm{ME}}\right)$ covered in this study include treatment income and basic expenditure subsidy, and the outpatient and inpatient medical expenses are included in the service function. The formula is as follows:

$$
\begin{aligned}
C_{\mathrm{ME}} & =\sum_{K=1}\left(C_{\mathrm{KINC}}+C_{\mathrm{KSUB}}\right) \\
& (K \text { means different types of medical institutions })
\end{aligned}
$$

where $C_{\mathrm{KINC}}$ represents the treatment income of various medical institutions, and $C_{\mathrm{KSUB}}$ represents the basic expenditure subsidy of various medical institutions.

To calculate treatment income, we used the following formula:

$$
C_{\mathrm{INC}}=C_{\mathrm{TINC}}\left(1-a_{\mathrm{p}} / a\right)
$$

where $C_{\text {TINC }}$ represents the actual total income of different medical institutions, $a$ represents the total income of sample institutions, $a_{\mathrm{p}}$ represents the total income of prevention services in the sample and $\left(1-a_{\mathrm{p}}\right) / a$ represents the proportion of treatment income of sample institutions to the total income of sample institutions after excluding prevention services.

The actual treatment income is expressed by $C_{\text {IINC, }}$ where $a_{i}$ represents the treatment income of the case in the sample, and $a_{i} /\left(a-a_{\mathrm{p}}\right)$ represents the proportion of the treatment income of the case in the sample after excluding the prevention service income. The formula is as follows:

$$
C_{\mathrm{IINC}}=\sum_{i}^{m} C_{\mathrm{INC}} a_{i} /\left(a-a_{\mathrm{p}}\right)
$$

The basic expenditure subsidy is allocated according to the proportion of service in the sample. $C_{\text {IsUB }}$ represents the total actual basic expenditure subsidy of all medical institutions, $C_{\text {TSub }}$ represents the total basic expenditure subsidy of various medical institutions, $C_{\mathrm{PSUB}}$ represents the basic expenditure subsidy of preventive services and $S$ represents the total amount of service in the sample and case services in the sample. The amount is expressed in $S_{i}$, and the amount of preventive services in the sample is expressed as $S_{\mathrm{p}}$. The formula is as follows:

$$
C_{\mathrm{ISUB}}=\sum_{i}^{m}\left[\left(C_{\mathrm{TSUB}}-C_{\mathrm{PSUB}}\right) s_{i} /\left(s-s_{\mathrm{p}}\right)\right]
$$

\section{Analysis of Factors Influencing Hospitalization Expenses for Diabetes}

Multiple linear regression modeling was used to analyze the hospitalization expenses and factors influencing diabetes, including age, sex, hospitalization time, operation, insurance payment type, medical institution type, among others, in order to determine the most important factors. In the multiple linear regression models, the hospitalization expenses of diabetic patients were consided to be dependent variables, the relative variable standard was 0.05 and the criterion for eliminating variables was 0.10 . The statistical analysis was conducted using SPSS version 20.0 software (IBM Corp., Armonk, NY, USA).

\section{Compliance with Ethics Guidelines}

All procedures performed in studies involving human participants were in accordance with the ethical standards of the Ethics Committee of Hainan Medical University and with the 1964 Helsinki declaration and its later amendments or comparable ethical standards. All informed consent forms and the data used were recorded and kept in accordance with guidelines by the Health Economics Association of Hainan Province. The informed consent forms and the data 
used were provided to the Ethics Committee of Hainan Medical University, which granted permission for the study.

\section{RESULTS}

\section{Medical Expenses for Management of Diabetes Patients and Influencing Factors}

The medical expenses due to the management of diabetes patients in Hainan Province can be classified as outpatient expenses and hospitalization expenses, with the outpatient expenses calculated to be 121.84 billion renminbi (RMB) and the hospitalization expenses calculated to be 120.87 billion RMB. The proportion of outpatient expenses and hospitalization expenses to the total medical costs of managing diabetes were 50.04 and $49.96 \%$, respectively. In 2016, the costs of drugs for the management of diabetes mellitus amounted to 94.35 billion RMB, accounting for $38.96 \%$ of the total treatment expenses for diabetes, of which $58.90 \%$ was in an outpatient setting and $41.10 \%$ was in a hospital setting.

The results of multiple stepwise regression analysis showed that $F=1089.99, p \leq 0.001$, thereby establishing the regression equation. From the standardized regression coefficient, the top three factors influencing hospitalization expenditure were the length of stay, type of insurance and age $(p<0.05)$ (Table 1$)$.

\section{The Institutional Flow of Medical Expenses}

According to the analysis of the flow direction of diabetes medical expenses in Hainan Province, the majority of patients' medical expenses flowed to the higher-level hospitals (general hospitals and hospitals of traditional Chinese medicine); this amounted to 198.47 billion $\mathrm{RMB}$, accounting for $81.95 \%$ of patients' total medical expenses. The proportion of drug charges in patients' costs in hospitals was $47.54 \%$, and the proportion of inspection charges was $24.01 \%$ (the proportion of drug and inspection charges in general hospitals was 46.83 and $21.51 \%$, respectively, and that in traditional Chinese hospitals was 31.65 and $29.56 \%$, respectively). A total of 35.62 billion RMB flowed to basic medical institutions (including township hospitals, community health service centers, village clinics and individual clinics), accounting for $14.71 \%$ of total patients' costs. In primary hospitals, the proportion of drug charges in patients' costs was $18.09 \%$, and the proportion of inspection charges was $8.27 \%$. Only a small proportion of the medical expenses was spent in outpatient service institutions and public health institutions (3.27 and 0.07\%, respectively; Fig. 1).

Table 1 Factors influencing medical expenses for the management of diabetes patients analyzed by multiple linear regression

\begin{tabular}{llllrrr}
\hline Model & \multicolumn{2}{l}{ Unstandardized coefficients } & & \multicolumn{2}{l}{ Standardized coefficient } & \\
\cline { 2 - 3 } & $\boldsymbol{B}$ & Standard error & & $\boldsymbol{B}$ & $\boldsymbol{t}$ statistic & $\boldsymbol{p}$ \\
\hline Constant & $11,588.107$ & 1370.894 & & & 8.453 & $<0.001$ \\
Length of stay & 802.009 & 13.069 & & 0.467 & 61.367 & $<0.001$ \\
Type of insurance & 3307.969 & 86.271 & 0.301 & 38.344 & $<0.001$ \\
Age & 96.727 & 3.083 & & 0.235 & 31.372 & $<0.001$ \\
Operation & $-16,321.107$ & 646.305 & & -0.181 & -25.253 & $<0.001$ \\
Hospital level & 2246.043 & 127.329 & & 0.155 & 17.64 & $<0.001$ \\
Type of hospital & -176.498 & 20.147 & & -0.076 & -8.761 & $<0.001$ \\
Sex & 650.588 & 128.961 & & 0.038 & 5.045 & $<0.001$ \\
\hline
\end{tabular}




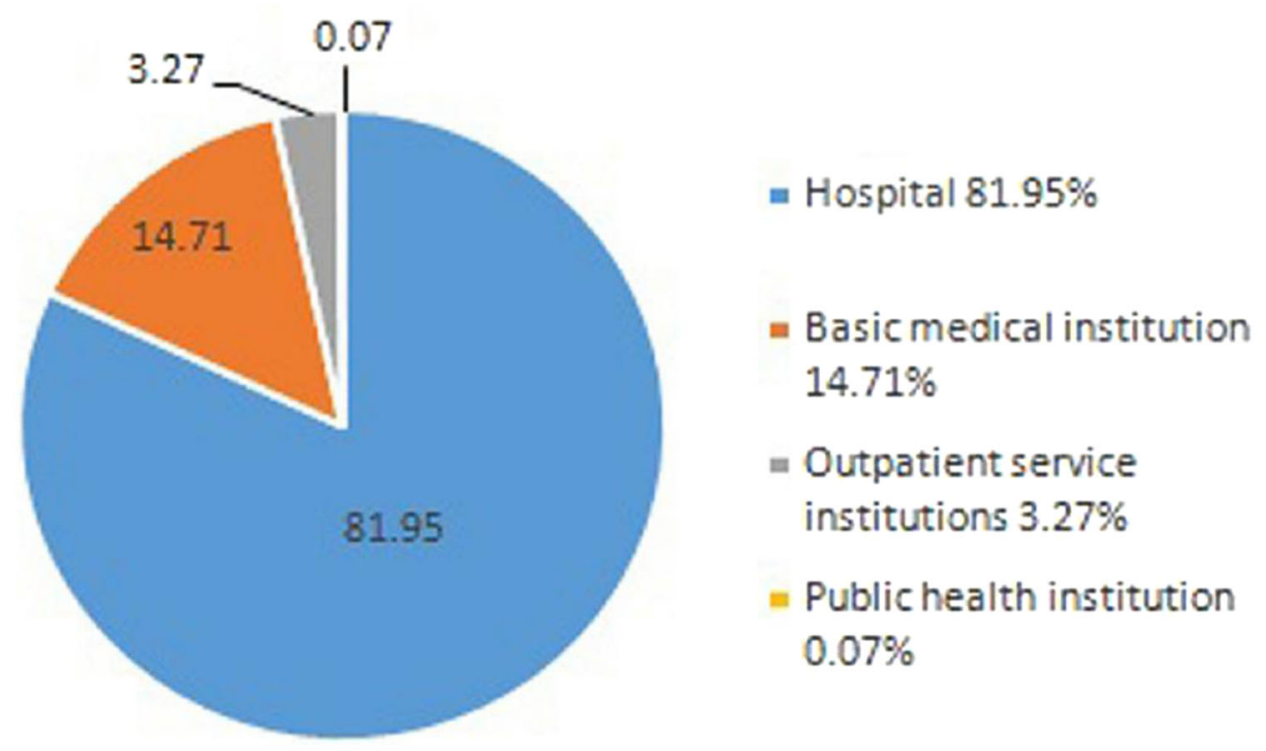

Fig. 1 The proportion of medical expenses according to medical institution type

\section{Composition of Service Functions of Different Medical Institutions}

According to our analysis of the composition of medical expenses for the management of diabetes mellitus in different types of medical institutions in Hainan Province, outpatient expenses accounted for $50.04 \%$ of medical costs and inpatient expenses accounted for $49.96 \%$. The total, outpatient and inpatient proportions of medical expenses for the management of diabetes mellitus and total costs according to different types of medical institutions in Hainan Province are shown in Fig. 2 and Table 2, respectively.

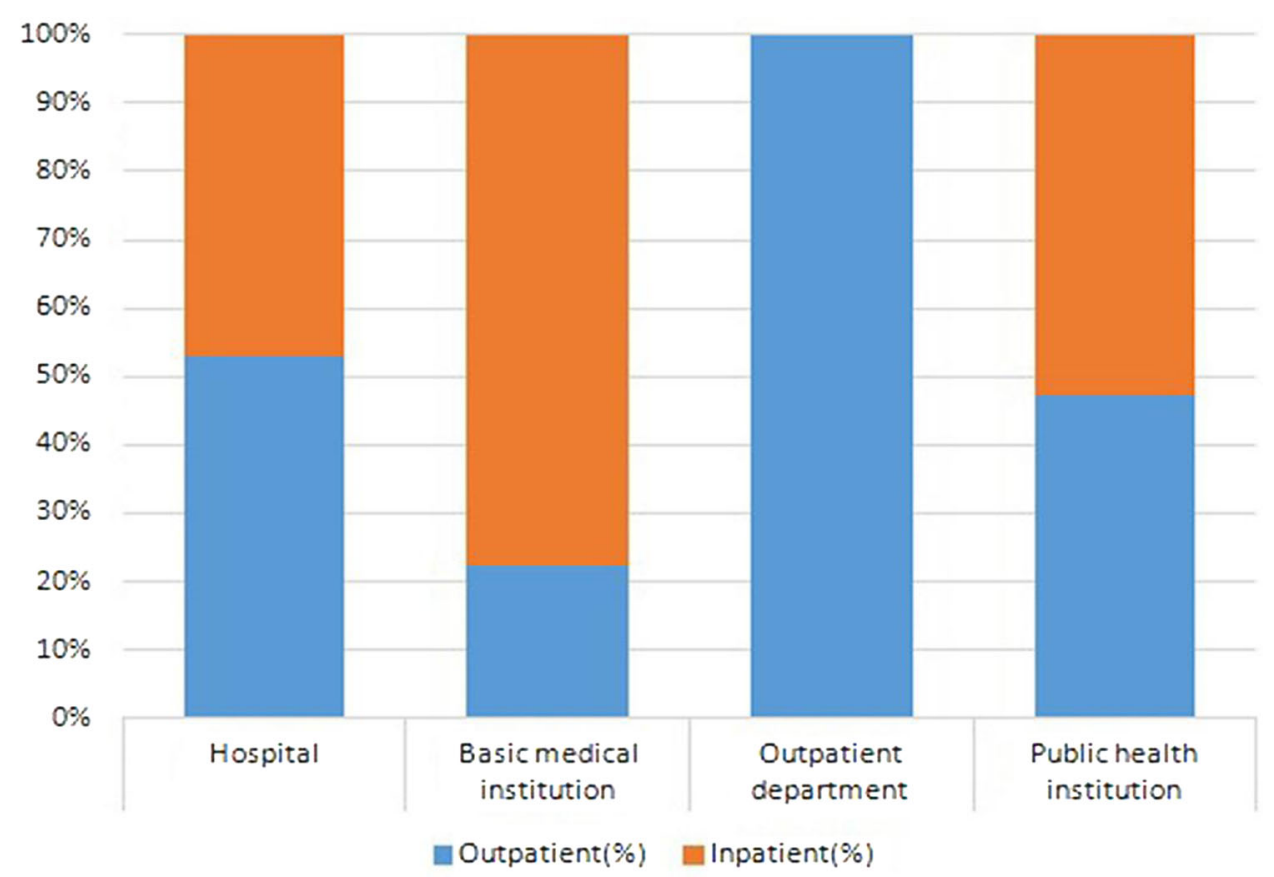

Fig. 2 Composition of service functions of different medical institutions 
Table 2 Allocation of medical expenses according to different levels of medical institution

\begin{tabular}{|c|c|c|c|c|c|c|}
\hline \multirow[t]{2}{*}{ Institution } & \multicolumn{2}{|l|}{ Total } & \multicolumn{2}{|c|}{ Outpatient setting } & \multicolumn{2}{|c|}{ Inpatient setting } \\
\hline & $\begin{array}{l}\text { Billion } \\
\text { RMB }\end{array}$ & $\begin{array}{l}\text { Proportion } \\
\text { (\%) }\end{array}$ & $\begin{array}{l}\text { Billion } \\
\text { RMB }\end{array}$ & $\begin{array}{l}\text { Proportion } \\
\text { (\%) }\end{array}$ & $\begin{array}{l}\text { Billion } \\
\text { RMB }\end{array}$ & $\begin{array}{l}\text { Proportion } \\
\text { (\%) }\end{array}$ \\
\hline Hospital & 198.4699 & 81.95 & 105.2187 & 86.83 & 93.2512 & 77.08 \\
\hline General hospital & 136.9160 & 56.54 & 91.2111 & 75.27 & 45.7049 & 37.78 \\
\hline $\begin{array}{l}\text { Traditional Chinese medicine } \\
\text { hospital }\end{array}$ & 61.5539 & 25.42 & 14.0077 & 11.56 & 47.5463 & 39.30 \\
\hline Basic medical institution & 35.6186 & 14.71 & 7.9716 & 6.58 & 27.6471 & 22.85 \\
\hline Outpatient department & 7.9142 & 3.27 & 7.9142 & 6.53 & - & - \\
\hline Public health institution & 0.1679 & 0.07 & 0.0792 & 0.07 & 0.0887 & 0.07 \\
\hline
\end{tabular}

$R M B$ Renminbi

Table 3 Age distribution of medical expenses for diabetes

\begin{tabular}{lll}
\hline $\begin{array}{l}\text { Age group } \\
\text { (years) }\end{array}$ & $\begin{array}{l}\text { Medical expenses } \\
\text { ratio (\%) }\end{array}$ & $\begin{array}{l}\text { Drug expenses } \\
\text { ratio (\%) }\end{array}$ \\
\hline $0-14$ & 4.74 & 3.6 \\
$15-29$ & 8.88 & 7.91 \\
$30-44$ & 14.5 & 11.51 \\
$45-59$ & 23.56 & 24.06 \\
$60-74$ & 29.99 & 31.13 \\
$\geq 75$ & 18.33 & 20.37 \\
\hline
\end{tabular}

Regarding outpatient expenses of the different institutions managing diabetes mellitus in Hainan Province, the proportion of primary medical and health institutions and outpatient institutions was determined to be $13.11 \%$. A total of $86.83 \%$ of outpatient expenses flowed to major hospitals. Based on the analysis of the inpatient expenses of diabetes, general hospitals and traditional Chinese hospitals accounted for 37.78 and $39.30 \%$ of total outpatient expenses, respectively.

\section{Distribution of Diabetes Treatment Expenses in Different Age Groups}

To further observe the role of age in expenses due to diabetes mellitus management and hospitalization, we divided diabetic patients aged $<75$ years into five age groups. We found that $71.88 \%$ of the people aged $>45$ years spent the largest proportion of their medical expenses on diabetes mellitus management; this was especially true for those aged 60-74 years for whom diabetes management accounted for $29.99 \%$ of total medical expenses (Table 3 ). The treatment cost of young and middle-aged diabetic patients aged 30-44 was also very high, accounting for $14.5 \%$ of total medical expenses. Of all drug expenses in Hainan Province, $75.56 \%$ were consumed by diabetic patients aged $>45$ years. The drug expenses of middleaged and elderly diabetic patients aged > 60 years exceeded half of the total expenditures for diabetic drugs. In 2016, the composition ratio of the drug costs of diabetes mellitus in Hainan Province was similar to that of the treatment expenses (Table 3).

\section{DISCUSSION}

The incidence of diabetes in China has increased rapidly in recent years, reaching 9.7\% of the general population in 2017 [9]. There are about 136 million diabetes patients worldwide, and diabetes has become a serious public health problem. There are many risk factors for diabetes, and the disease is a life-long condition. With disease progression, patients may develop 
any number of a variety of severe complications, such as blindness, kidney failure and gangrene. In China, $73.2 \%$ of patients with diabetes suffer from chronic complications, and the treatment costs of these complications account for $>80 \%$ of the total medical costs of patients with diabetes. Among these chronic complications, cardiovascular disease, cerebrovascular disease and kidney disease are the main causes of the disability and death of patients with diabetes. The medical expenses generated by this disease are also the most important part of the total expenses in China [10]. The medical expense burden of diabetes patients is so heavy that in 2016 the total medical expenses added up to 100 billion RMB, accounting for $5 \%$ of the medical expenses for all chronic non-communicable diseases. The out-of-pocket costs accounted for 30\% of the total expense. Diabetes mellitus places tremendous financial pressure on Chinese society and on the individual patient's family. The risk of a diabetic patient's family falling into poverty is high [11].

In our study, the medical expenses for managing diabetes in Hainan Province added up to 242.17 billion RMB, accounting for $28.3 \%$ of the total medical expenses for managing endocrine, nutritional and metabolic diseases ( 855.71 billion RMB). This suggests that government and health institutions should pay more attention to the financial burden of diabetes and implement additional measures to control the medical expenses. We also found that in terms of diabetes management, the medical and drug expenses for outpatients are higher than those for inpatients. One reason is that patients prefer outpatient treatment and therefore spend more on drug expenses than inpatients because this incurable chronic disease requires long-term medication. Drugs, diagnosis and treatment costs are long-term burdens for patients with chronic diseases [12]. Cost ratios between people with and without diabetes have been reported to range from 1.5 to 4.4 , which is different from cost ratios in other diseases [13-15]. Jacobs et al. found the largest differences in health expenses came from prescribed medication in pharmacies (cost ratio diabetes:no diabetes: 2.2) [16].
We found that the age and sex of the patient, number of days hospitalized, type of operation, type of medical insurance payment, type of medical institution, level of medical institution and hospitalization expenses had a significant impact on the total medical expense, in accordance with results reported previously [17]. The medical cost of surgical patients is significantly higher than that of other patients, which may be a direct result of the severity of the disease and the higher cost of treatment. Medical insurance has been found to have the potential to promote an increase in medical expenses, with health insurance impacting on a patient's ability to pay and a doctor's behavior regarding diagnosis and treatment [18]. In our study, we reached the same conclusion. One explanation is that patients reimbursed by medical insurance can afford more treatment than self-paid patients. The former prefer high-tech, highly effective therapies and expensive drugs when choosing treatment methods and means, so their costs are likewise higher than those of selfpaid patients. The longer the hospital stay per capita, the higher the cost of treatment. Compared with non-surgical patients, surgical patients stay in the hospital longer, and medical expenses naturally increase. The medical expenses of patients with diabetes mellitus and associated conditions are considerably higher than those of other patients, which may be directly related to the severity of the disease and the cost of treatment [19].

In terms of overall cost, hospital expenditure occupies the dominant position, with drug and inspection charges in hospitals being higher than those of other institutions. The drug and inspection charges of primary institutions are $<$ $20 \%$ of total medical expenditure, possibly due to patients' trust in hospitals being higher than that in primary healthcare institutions. Due to the serious shortage of medical technical personnel at the primary care level, patients cannot get access to a superior hospital for further study for a long time, and it would appear that the professional level of these personnel cannot be timely and effectively improved. At the same time, the serious shortage of funds in medical institutions providing primary care is a common social phenomenon, Therefore, the 
technical staff and equipment of primary institutions cannot meet the needs of patients, so they cannot gain the trust of "patients with minor diseases." As a result, the outpatient service and inpatient visits of grassroot institutions are seriously insufficient, which leads to various drug and inspection charges being fewer and lower $[20,21]$. The acceleration of urbanization and the lag in providing basic health facilities to meet demand means that high-quality medical resources are concentrated in large general hospitals [22]. Consequently, Chinese patients prefer hospitals for better treatments, a preference that is related to the functional orientation, diagnosis and treatment capacity of such institutions. Overall, the role of primary healthcare institutions in the treatment of diabetes needs to be further strengthened [23]. According to the policy of graded diagnosis and treatment in China, especially the prevention of diabetes, the management of diabetes patients, the prognosis of disease, the rehabilitation of primary medical and health institutions and the role of medical and health institutions at all levels can be improved. The present state is one of the reasons why the economic burden of diabetes is so heavy.

Regarding the service function of medical expenses of diabetes, there is a large difference between Hainan Province and the whole country, with the costs of hospitalization for diabetes in Hainan Province being about ten percentage points higher than the overall national level. This may be due to the high prevalence of diabetes mellitus in Hainan Province and the longterm hospitalization required. An analysis of the different types of medical institutions revealed that most expenses are spent in outpatient costs and drugs. In Ulrich et al.'s study, diabetes patients with insulin monotherapy or with a combination of insulin and oral antidiabetic drugs had significantly elevated costs compared with individuals without diabetes (3.98 vs. 2.58 , respectively) [24].

Diabetes mellitus is common among the elderly: the higher the age, the higher the medical expense [25]. Our research showed that the hospitalization expenses of patients aged $<$ 75 years old increased with age. However, in the final analysis, it is not age itself that was found to cause elevated medical expenses; rather, the decline of the functions of the human immune system and organs play a role. A decline of organ functions translates into more basic diseases; consequently, elderly diabetic patients may have one or more complications and need more care as the disease progresses, resulting in higher costs than patients in other age groups [26]. In recent years, however, there has been a trend for diabetic patients to be younger, attributed in part to the large changes in lifestyle and the living environment, greater working pressure, disordered dietary structure and irregular lifestyle that have appeared in the last decades. In comparison to older patients, patients in the younger age groups have short disease onset time, relatively single-treatment indicators, fewer complications and lower treatment costs. Thus, the results of our age distribution analysis gives a clear indication that people in the age group associated with high expenses should pay great attention to the prevention of diabetes.

\section{Suggestion to Policy-Makers}

\section{Controlling Hospitalization Expenses and Improving Medical Insurance}

Studies on hospitalization costs for the management of diabetes mellitus and other diseases have shown that the number of hospitalization days are a positive correlation factor affecting hospitalization costs and that, consequently, limiting hospitalization days is an effective way to control medical costs [27]. Diabetic patients can reduce hospitalization rates by regulating their diet to control blood sugar, taking medicine on time and undergoing regular examinations to reduce complications.

The medical insurance management department should improve the level of medical insurance supervision and management, monitor the expenditure of hospitalization expenses for patients with different types of medical insurance, ensuring it is kept within a reasonable range, ensure the rational use of the funds available and regulate the unreasonable increase in hospitalization expenses. Reimbursement to the outpatient is still too low, 
resulting in large out-of-pocket expense. Therefore, the medical insurance management department should also pay more attention to this reimbursement ratio and make some adjustments.

\section{Using Hierarchical Diagnosis and the Treatment System to Control Medical Expense}

Medical expenses for diabetes in Hainan Province were mainly in high-level medical institutions, and there is little patient flow to primary healthcare institutions. Therefore, to reduce the economic burden of disease, it is necessary to improve both the role of primary healthcare institutions in the treatment of diabetes and the healthcare system so that patients receive good advice in choosing a primary healthcare institution [28]. Healthcare management institutions should strengthen the vocational skills training of medical staff in primary institutions, dispatch doctors from higher hospitals to attend clinics at the primary care level and improve the ability of healthcare workers at the grassroots level to manage chronic diseases similar to diabetes. The government should attach importance to the construction of basic facilities and support and encourage primary care medical institutions to carry out public health services, with the aim to increase the rate of patients visiting primary healthcare institutions.

\section{Strengthen Prevention Measures and Curb the Prevalence of Diabetes}

In the early stage of diabetes, strict control of blood glucose is an effective measure to reduce and delay microvascular complications $[29,30]$. The American Diabetes Association published "Diabetes Medical Standards", which recommends that adult diabetes screening should start at age 45 years [31]. Basic medical institutions provide comprehensive preventive care for elderly patients with diabetes, including lectures on diabetes that provide patients with knowledge of the disease that will enable them to better interpret and communicate their condition and interpret the problems they may encounter, and conducting research on the patient's daily diet, exercise habits, blood sugar monitoring, and drug use. Specific preventive and healthcare measures can be formulated according to each patient's condition in terms of lifestyle changes, exercise and diet regimens, massage, among others. Nursing staff should regularly conduct telephone return visits and family return visits and establish online communication groups to solve the problems encountered by patients in real-time. In this way, patients can be encouraged to maintain good living habits in terms of diet, exercise and lifestyle [32].

\section{Study Limitations}

Diabetes on its own is associated with high healthcare costs, but complications of the eye, kidney and cardiovascular and nervous systems may also arise, and these significantly increase the medical expenses. In this study we adopted SHA 2011, an internationally accepted healthcare accounting method, to study only the costs associated with the primary diagnosis and treatment of diabetes; the complications of diabetes were not included in the calculation. Future studies will explore the costs related to diabetes and complications. We only researched the direct financial burden of medical expenses for treating diabetic patients; any indirect cost burdens were not considered. In future studies, we will continue to explore the indirect burden accounting method so as to more accurately assess the economic pressures placed on diabetes patients.

\section{CONCLUSION}

This study on the medical expense of diabetes patients in Hainan province revealed that the economic burden of health expenses of diabetes patients is relatively heavy. Our study emphasizes the importance of reducing the economic burden of Hainan residents and of identifying the main factors which influence the medical expenses. We suggest promoting the hierarchical diagnosis and treatment system, improving the reimbursement ratio of medical insurance, 
perfecting drug policies and strengthening prevention policies.

\section{ACKNOWLEDGEMENTS}

The authors express thanks to China National Health Development Research Center for their help with SHA 2011 analysis. We also gratefully acknowledge the support of Hainan provincial health commission in providing data. We also would like to thank all the participants in this study.

Funding. This work was supported by National Natural Science Foundation of China (71673299) and the Social Science Fund Project Planning of Shenyang City (18014). The Rapid Service Fee was funded by the authors.

Authorship. All named authors meet the International Committee of Medical Journal Editors (ICMJE) criteria for authorship for this article, take responsibility for the integrity of the work as a whole, and have given their approval for this version to be published.

Authorship Contributions. XW designed the study, helped in implementing the project and reviewed the manuscript. YD and CL were the main drafters of the manuscript. YD was responsible for the revision of the manuscript. $\mathrm{PZ}$ and YZ did some of the expeditionary research. QT and SW contributed to the coordination and manuscript editing. All authors participated in the analysis and discussion of the research, under the leadership and instructions of XW. All authors read and approved the final manuscript.

Disclosures. Yuanyuan Dong, Chunping Liu, Peng Zhou, Yalan Zhu, Qingcheng Tang, Siyu Wang and Xin Wang have nothing to disclose.

Compliance with Ethics Guidelines. All procedures performed in studies involving human participants were in accordance with the ethical standards of the Ethics Committee of Hainan Medical University and with the 1964 Helsinki declaration and its later amendments or comparable ethical standards. All informed consent forms and the data used were recorded and kept in accordance with guidelines by the Health Economics Association of Hainan Province. The informed consent forms and the data used were provided to the Ethics Committee of Hainan Medical University, which granted permission for the study.

Data Availability. The datasets generated during and/or analysed during the current study are available from the corresponding author on reasonable request.

Open Access. This article is distributed under the terms of the Creative Commons Attribution-NonCommercial 4.0 International License (http://creativecommons.org/licenses/ by-nc/4.0/), which permits any noncommercial use, distribution, and reproduction in any medium, provided you give appropriate credit to the original author(s) and the source, provide a link to the Creative Commons license, and indicate if changes were made.

\section{REFERENCES}

1. Dall TM, Yang W, Gillespie K, et al. The economic burden of elevated blood glucose levels in 2017: diagnosed and undiagnosed diabetes, gestational diabetes, and prediabetes. Diabetes Care. 2019;42(10):dc181226. https://doi.org/10.2337/ dc18-1226.

2. Jing Z, Chu J, Imam Syeda Z, et al. Catastrophic health expenditure among type 2 diabetes mellitus patients: a province-wide study in Shandong, China. J Diabetes Investig. 2019;10(2):283-9.

3. International Diabetes Federation. IDF diabetes atlas, 7 th edition. Online version of IDF diabetes atlas. 2015. http://www.diabetesatlas.org.

4. Wang L, Gao P, Zhang M, et al. Prevalence and ethnic pattern of diabetes and prediabetes in China in 2013. JAMA. 2017;317(24):2515-23. 
5. King H, Aubert RE, Herman WH. Global burden of diabetes, 1995-2025: prevalence, numerical estimates, and projections. Diabetes Care. 1998;21(9):1414-31.

6. Zhichao Z. Study on age distribution characteristics and related factors of diabetes mellitus in young patients. Med Forum. 2018;10:1304-5.

7. Qixin D, Hui W, Huimei M, Xuanxuan W, Shangcheng Z, Xiang Z. Analysis of influencing factors of economic burden of diseases among elderly patients with common chronic diseases in Haikou City. Med Soc. 2019;6:39-42.

8. OECD, Eurostat, WHO. A system of health accounts 2011-revised edition. Paris: OECD Publishing; 2011.

9. Yang W, Lu J, Weng J, et al. Prevalence of diabetes among men and women in China. N Engl J Med. 2010;362(12):1090-101.

10. He X, Zhang Y, Ruan Z, Li L, Wu J. The prevalence and related direct medical costs of chronic complications among patients with type 2 diabetes in China. Value Health. 2018;21:S37.

11. Yuhui Z, Quan W, Peipei C, Feng G, Xiufeng W. The medical costs and financing burden of diabetes mellitus in China. Chin Health Econ. 2017;36(4):17-9.

12. Zongjie L, Wenfang C, Wenwen Z, Caijun Y, Shimin Y, Yu F. Medication analysis of hypertension and diabetes mellitus in primary medical institutions in Shaanxi Province in 2016. Chin Pharm Matters. 2017;12:1497-505.

13. Ng CS, Lee JY, Toh MP, Ko Y. Cost-of-illness studies of diabetes mellitus: a systematic review. Diabetes Res Clin Pract. 2014;105(2):151-63.

14. Rosella L, Lebenbaum M, Fitzpatrick T, et al. Impact of diabetes on healthcare costs in a populationbased cohort: a cost analysis. Diabetic Med. 2016;33(3):395-403.

15. Sortsoe C, Green A, Jensen PB, Emneus M. Societal costs of diabetes mellitus in Denmark. Diabet Med. 2016;33(7):877-85.

16. Jacobs E, Hoyer A, Brinks R, Icks A, Kuß O, Rathmann W. Healthcare costs of type 2 diabetes in Germany. Diabet Med. 2017;34(6):855-61.

17. Cao P, Wang $\mathrm{K}$, Zhang $\mathrm{H}$, Zhao R, Li C. Factors influencing the hospitalization costs of patients with type 2 diabetes. Asia Pacif J Public Health. 2015;27[Suppl 2]:55S-60S.
18. Yang C, Huang Z, Sun K, Hu Y, Bao X. Comparing the economic burden of type 2 diabetes mellitus patients with and without medical insurance: a cross-sectional study in China. Med Sci Monit. 2018;24:3098-102.

19. Bao X, Yang C, Fang K, Shi M, Yu G, Hu Y. Hospitalization costs and complications in hospitalized patients with type 2 diabetes mellitus in Beijing, China. J Diabetes. 2017;9(4):405-11.

20. Xianwen L. Consideration on related issues of grading treatment model. Health Econ Res. $2015 ; 3: 18-20$

21. Zhu S. Building talented workforce teams is the key to strengthened primary care. Chin J General Practitioners. 2016;15(8):599-600.

22. Wang Y, Sun L, Hou J. Hierarchical medical system based on big data and Mobile internet: a new strategic choice in health care. JMIR Med Inform. 2017;5(3):e22. https://doi.org/10.2196/medinform. 6799.

23. Hongwei T. Community outpatient expenses and management of key population with type 2 diabetes mellitus Physical analysis. China Maternal Child Health Study. 2017;28[S1]:682-3.

24. Ulrich S, Holle R, Wacker $\mathrm{M}$, et al. Cost burden of type 2 diabetes in Germany: results from the population-based KORA studies. BMJ Open. 2016;6(11):e012527.

25. Yang Y, Goh SY, Tan SB, et al. The burden of diabetes mellitus in elderly patients from an Asian tertiary hospital. Eur J Internal Med. 2012;23(1):e1-4.

26. Jiarong D, Lu L, Wantao D. Analysis of influencing factors of hospitalization costs for diabetic patients in a hospital. Chongqing Med. 2018;47(29):3773-5.

27. Yaling D, Zhong W. Multivariate regression analysis of influencing factors on hospitalized intensive treatment of type 2 diabetes mellitus. China Med Records. 2016;17(8):49-52.

28. Lingzhi Z. Experience and revelation from pilot trials of first and two-way referral in urban communities in China. Chongqing Med. 2010;39(2):250-1.

29. Zoungas S, De Galan BE, Ninomiya T, et al. Combined effects of routine blood pressure lowering and intensive glucose control on macrovascular and microvascular outcomes in patients with type 2 diabetes: new results from the ADVANCE trial. Diabetes Care. 2009;32(11):2068-74. 
30. ADVANCE Collaborative Group, Patel A, MacMahon $\mathrm{S}$, et al. Intensive blood glucose control and vascular outcomes in patients with type 2 diabetes. N Engl J Med. 2008;358(24):2560-72.

31. [No authors listed]. Standards of medical care in diabetes-2016: summary of revisions. Diabetes Care. 2016;39[Suppl 1]:S4-5.
32. Yiwei G, Pu Z, Jiuhong M, et al. Effects of integrated intervention of community traditional Chinese medicine on diabetes patients. Shanxi J Med. 2019;48(15):1852-3. 